of stay. In other ways, for some other reasons, especially in our national government insurance, we could not perform those procedures in tertiary hospital as a routine procedure.

Methods In this retrospective study, we would evaluate, the length of stay difference between open conventional and laparoscopy cholecystectomy on calculous cholecystitis patients from January until December 2017. The length of stay after these procedures would be recorded. All patients had the same prophylactic antibiotic (2 gram of Cefazolin iv) and 1 gram Paracetamol iv twice daily. The patients who have been done the conversion to open cholecystectomy would be excluded from this study.

Results 94 calculous cholecystitis cases were evaluated in this study during 1 year period. 71 cases have been done open conventional cholecystectomy, 23 cases have been performed laparoscopy cholecystectomy. The mean length of stay on the open arm was 1.7 days, and 1.5 days for laparoscopy cholecystectomy. 3 patients on the open conventional arm had biliary pancreatitis and empyema gall bladder, and that case has a longer length of stay (3 days). There was no post-operative complication recorded.

Conclusions There was no length of stay difference between open conventional and laparoscopy cholecystectomy on calculous cholecystitis patients.

\section{IDDF2020-ABS-0084 COMPARISON OF 1L ADJUVANT AUXILIARY PREPARATIONS WITH 2L SOLELY POLYETHYLENE GLYCOL PLUS ASCORBIC ACID REGIME FOR BOWEL CLEANING: A META-ANALYSIS OF RANDOMIZED, CONTROLLED TRIALS}

${ }^{1}$ Xin Yuan*, 'Zhixin Zhang, ${ }^{1}$ Jiarong Xie, ${ }^{2} Y u$ Zhang, ${ }^{3}$ Lu Xu, ${ }^{2}$ Weihong Wang, ${ }^{2}$ Lei Xu. ${ }^{1}$ College of Medicine, Ningbo University, China; ${ }^{2}$ Department of Gastroenterology, Ningbo First Hospital, China; ${ }^{3}$ Clinical Department for Intensive Care, Ningbo Second Hospital, China

\subsection{6/gutjnl-2020-IDDF.68}

Background The effectiveness of additional usage of adjuvants for bowel preparation is still unclear. This study compared $1 \mathrm{~L}$ polyethylene glycol plus ascorbic acid with adjuvant drug regimens (1L-PEG-AA, lower-volume) with $2 \mathrm{~L}$ polyethylene glycol plus ascorbic acid (2L-PEG-A, low-volume) to evaluate whether the adjuvants can be used to reduce the standard dosage of purgative further.

Methods The PubMed/MEDLINE, EMBASE, Cochrane Library and Web of Science database were searched up to March 2020 for randomized controlled trials (RCTs). The primary outcome was the efficacy of bowel preparation, and the secondary outcomes were patients' tolerability and complication rate. The relative risk (RR) and mean difference (MD) with 95\% confidence intervals (CI) were pooled for dichotomous and continuous variables separately. The overall quality of evidence was assessed using the GRADEpro guideline development tool.

Results Five RCTs with a total of 1013 patients were included, and the majority of patients were outpatients from different hospitals. The pooled data showed no significant difference in the adequate bowel preparation rate $(89.3 \%$ versus $89.4 \%$, RR 1, 95\%CI $0.95-1.05, \mathrm{I}^{2}=47 \%$ ) (figure $1 \mathrm{~A}$ ) as well as in the complication rate (RR for nausea $1.22,95 \% \mathrm{CI}$ $0.89-1.65, \mathrm{I}^{2}=49 \%$; RR for bloating 0.96 , 95\%CI $0.73-1.28$, $\mathrm{I}^{2}=0 \%$; RR for vomiting 0.69 , 95\% CI $0.32-1.50, \mathrm{I}^{2}=33 \%$;

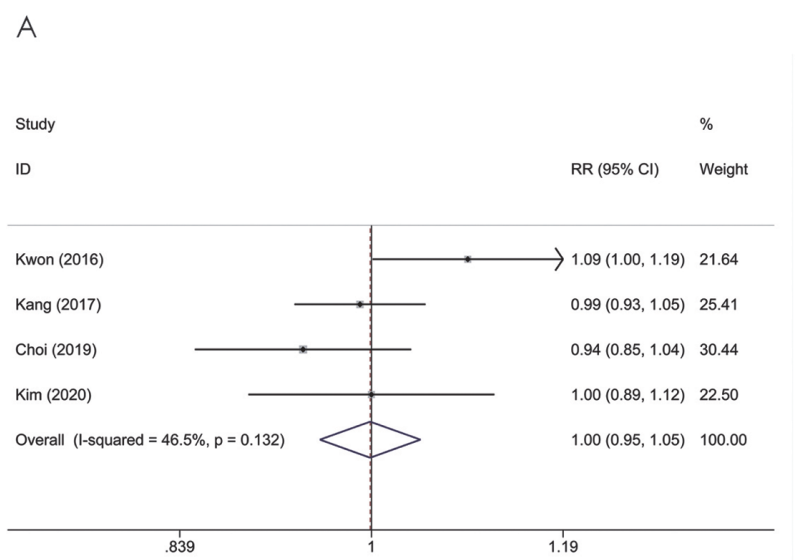

Abstract IDDF2020-ABS-0084 Figure 1A Forest plot on the efficacy and willingness between 1L-PEG-AA and 2L-PEG-A

B

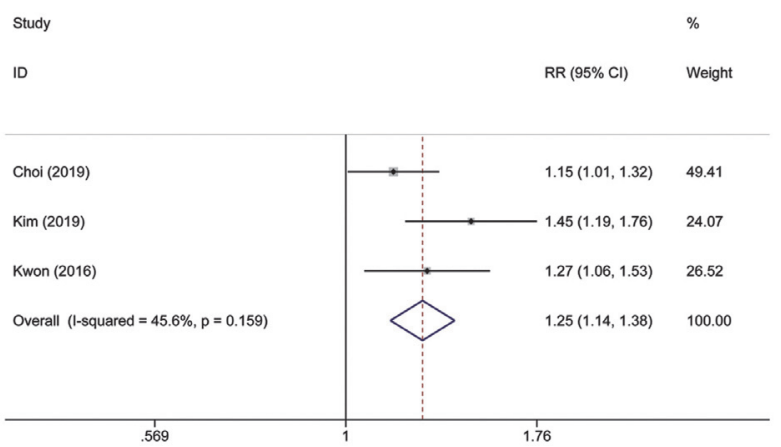

Abstract IDDF2020-ABS-0084 Figure 1B Forest plot on the efficacy and willingness between 1L-PEG-AA and 2L-PEG-A

RR for abdominal pain 1.01, 95\%CI 0.61-1.69, $\mathrm{I}^{2}=0 \%$ ). But a significantly higher willingness rate was observed in the lower-volume $(85.1 \%$ versus $67.9 \%$, RR 1.25 , 95\%CI 1.14 $1.38, \mathrm{I}^{2}=46 \%$ ) (figure $1 \mathrm{~B}$ ). The quality of primary outcome evidence was moderate.

Conclusions The findings of this meta-analysis revealed that 1L-PEG-AA might be a viable alternative to 2L-PEG-A, with comparable effectiveness, better patient preference, and no statistically significant adverse event occurrence.

\section{IDDF2020-ABS-0085 UNDERWATER VERSUS CONVENTIONAL ENDOSCOPIC MUCOSAL RESECTION FOR SMALL SIZE NON-PEDUNCULATED COLORECTAL POLYPS: A RANDOMIZED CONTROLLED TRIAL}

${ }^{1}$ Zhixin Zhang ${ }^{*},{ }^{2}$ Yonghong Xia, ${ }^{3}$ Hongyao Cui, ${ }^{1}$ Xin Yuan, ${ }^{4}$ Chunnian Wang, ${ }^{1}$ Jiarong Xie, ${ }^{2}$ Yarong Tong, ${ }^{5}$ Weihong Wang, ${ }^{5}$ Lei Xu. ${ }^{1}$ College of Medicine, Ningbo University, China; ${ }^{2}$ Department of Gastroenterology, Ninghai Second Hospital, China; ${ }^{3}$ Department of Gastroenterology, Haishu Second Hospital, China; ${ }^{4}$ Ningbo Clinical and Pathological Diagnosis Center, China; ${ }^{5}$ Department of Gastroenterology, Ningbo First Hospital, China

\subsection{6/gutjnl-2020-IDDF.69}

Background Underwater endoscopic mucosal resection (UEMR) is a recently developed technique and is performed during 